\title{
L'anticorps anti-LKM, est dirigé contre un antigène du réticulum endoplasmique hépatocytaire
}

\author{
Localisation de l'antigène $L K M_{1}$ dans le foie
}

Deux maladies du foie sont associées à la présence dans le sérum des patients d'anticorps antimicrosomes : l'hépatite chronique active auto-immune et l'hépatite due à un diurétique, l'acide tiénilique (Diflurex ${ }^{\circledR}$ ). L'hépatite chronique active auto-immune de l'enfant peut être classée en deux groupes : dans l'un de ces groupes, le sérum contient un anticorps anti-muscle lisse, dans l'autre un anticorps antimicrosomes appelé anti-LKM (liver-kidney microsomes 1). Au cours de l'hépatite due à l'acide tiénilique, le sérum contient un autre anticorps anti-microsomes, appelé anti-LKM $M_{2}$. L'antigène correspondant à l'anticorps anti-LKM $\mathrm{KM}_{2}$ a été identifié : il s'agit probablement d'un constituant du cytochrome P-450. La localisation de l'antigène correspondant à l'anti$\mathrm{LKM}_{1}$ a été récemment précisée [1]. En utilisant la technique du Western blot sur des fractions purifiées du réticulum endoplasmique lisse et rugueux, il a été montré que l'anticorps anti-LKM, présent dans le sérum de patients reconnaissait une protéine de $50 \mathrm{kDa}$ de ces deux organites. La protéine était absente des mitochondries, des membranes lysosomales, de la membrane plasmique et des ribosomes. Par immunomarquage à l'or radioactif et par immunoperoxydase indirecte, l'antigène a pu être localisé sur des coupes de foie : les sites antigéniques sont exclusivement situés à la face cytoplasmique du réticulum endoplasmique lisse et rugueux. Aucun marquage n'a été observé sur l'appareil de Golgi, les peroxysomes, les mitochondries, les lysosomes, la membrane plasmique ou les ribosomes. De plus, le marquage a été observé exclusivement dans les hépatocytes, et jamais dans les cellules de Küpffer ni dans les cellules endothéliales.

L'anticorps anti-LKM $\mathrm{L}_{1}$ peut donc être considéré comme un marqueur spécifique du réticulum endoplasmique hépatocytaire. L'antigène reste toutefois à identifier.

S.E.

1. De Lemos-Charandini C, Alvarez F, Bernard O, Homberg JC, Kreibich G. Anti-liverkidney microsome antibody is a marker for the rat hepatocyte endoplasmic reticulum. Hepatology $1987 ; 7$ : 468-75.

\section{口曰 BRÈVES}

Les androgènes peuvent modifier la morphologie de neurones du système nerveux central même chez l'adulte. Le modèle le mieux connu est celui des muscles bulbo-caverneux et levator ani (BCLA) du rat. Ces muscles, attachés à la base du pénis, sont innervés par des neurones originaires de la moelle lombaire. A la naissance, le nombre de neurones destinés à cette zone diffère peu entre les deux sexes. Dans les jours qui suivent, la perte de neurones est beaucoup plus élevée chez la femelle que chez le mâle. A l'âge adulte, il reste à la femelle environ 60 petits neurones innervant le sphincter neurones destinés au sphincter anal et aux muscles BCLA. Il a été reconnu récemment que les androgènes peuvent exercer leur action neurotrope chez l'adulte. Kurz et al. [1] ont pu montrer que la longueur des dendrites du noyau spinal correspondant diminue de moitié après castration et est ramenée à la normale par la testostérone. Forger et Breedlove [2] ont observé que chez les souris à pattes blanches, qui se reproduisent seulement au printemps et en été, non seulement la taille des gonades, mais aussi celle des corps cellulaires des neurones correspondants, varient parallèlement à la longueur du jour. Il apparaît ainsi que la distinction entre actions organisationnelles et activatrices des stéroïdes sexuels sur le système nerveux central est trop tranchée, et que ces stéroïdes peuvent exercer des influences de longue durée, en particulier saisonnières. On constate, de plus, qu'un circuit neural qui n'est pas utilisé s'atrophie non seulement fonctionnellement mais aussi physiquement, et qu'il peut croître à nouveau s'il redevient nécessaire [3].

[1. Kurz EM, et al. Science 1986, 232: 395-8.

[2. Forger NG, Breedlove SM.J Neurobiol 1987 ; 18 : 155-65.]

[3. De Voogd'TJ. Trends Neurol Sci 1987 ; 10 ; 341-2.] 
口 L'uromoduline est un immuno-suppresseur ayant de l'affinité pour l'interleukine 1 (IL-1). Cette protéine, identique à la "glycoprotéine de TammHorsfall " a été initialement purifiée à partir de l'urine de femme enceinte. Son ADN complémentaire a été récemment isolé, permettant la déduction de la séquence protéique et l'analyse de l'expression du gène. Seul le rein synthétise le messager de l'uromoduline qui est particulièrement abondante au niveau de la partie large du segment ascendant de l'anse de Henlé. La protéine lie, avec une forte affinité, au niveau du même site (ou de sites chevauchants), l'IL-1 et le TNF (tumor necrosis factor). Elle pourrait donc jouer un rôle important d'immuno-modulateur en contrôlant la concentration en formes libres de lymphokines circulantes. [Hession C, et al. Science 1987 ; 237 : 1479-84.] $\square \square$ Les agonistes $\alpha$-adrénergiques peuvent augmenter la concentration intracellulaire de calcium $\left(\mathrm{Ca}^{++}\right)$par deux mécanismes. Le premier est la synthèse d'inositol triphosphate conduisant à la libération de $\mathrm{Ca}^{++}$stocké dans les citernes du réticulum endoplasmique; le deuxième est l'ouverture de canaux calciques de la membrane plasmique. Ces deux réponses pourraient dépendre de deux types pharmacologiquement distincts de récepteurs.

[Hanb C, et al. Nature 1987 ; 329: 333-5.]

Das neurones et des cellules gliales de la rétine peuvent avoir un progéniteur commun tout au long du développement comme cela vient d'être montré très élégamment. Afin de marquer un clone cellulaire, un vecteur rétroviral portant le gène d'Escherichia coli codant pour la $\beta$ galactosidase a été injecté entre l'épithélium et la rétine chez des rats nouveau-nés, c'est-à-dire à un stade très tardif du développement rétinien. Les cellules proliférant à ce stade ont incorporé et transmis le gène et les clones cellulaires l'exprimant ont été retrouvés chez l'animal adulte. Ces clones présentent dans certains cas une hétérogénéité remarquable, comprenant notamment neurones et cellules gliales. Outre son aspect technique novateur, cette étude pose sous un angle nouveau le problème de la fonction de la cellule gliale. Considérée encore très récemment comme une simple cellule de soutien, elle acquiert en effet aujourd'hui le statut de frère jumeau de l'élément le plus noble du cerveau, le neurone.

[Turner DL, Cepko CL. Nature 328 ; 1987 : 131-6.]

\section{Virus HTLV-I et neurofibromatose}

Le gène tat du virus HTLV-I (human lymphotropic virus type I) est un oncogène et induit des tumeurs neurofibromateuses. Des animaux transgéniques ayant intégré une construction comportant le gène tat,$d u$ virus HTLV-I sous le contrôle du LTR (long terminal repeat) de ce virus ont été créés. Le gène tat code pour une protéine "trans-activatrice ", c'est-à-dire augmentant l'activité transcriptionnelle de son propre promoteur et, peut-être également, de promoteurs cellulaires. Deux phénotypes ont été observés chez les souris qui expriment le transgène [1] : un phénotype "petit ", comportant une aplasie thymique et une hypotrophie globale, qui est associè à une mortalité précoce (dans les trois premières semaines). Ces animaux transcrivent activement le transgène dans le thymus et d'autres tissus, dont le muscle. Le second phénotype, "grand ", est caractérisé par l'apparition, après la $m / s n^{\circ} 10$ vol. 3, décembre 87 sixième semaine de vie, de multiples tumeurs neurofibromateuses, le plus souvent bénignes. Le développement et la taille de ces souris transgéniques sont par ailleurs normaux. Dans ce cas, le transgène est peu ou pas exprimé dans le thymus et est très actif dans d'autres tissus, surtout le muscle.

Les deux phénotypes coexistant dans la descendance d'un même fondateur transgénique, les mécanismes de leur création restent intrigants. Il est possible que les fondateurs aient été des mosaïques pour plusieurs sites chromosomiques d'intégration, les phénotypes des descendants correspondant alors à la ségrégation indépendante de ces trangènes intégrés en des sites différents. La nature neurofibromateuse des tumeurs évoque les lésions de la maladie de von Recklinghausen [2] dont il existe des formes sporadiques, les formes génétiques étant liées à un locus du chromosome $17\left(\mathrm{~m} / \mathrm{s} \mathrm{n}^{\circ} 7\right.$, vol. 3, p. 433).
Par ailleurs, HTLV-I ayant été impliqué dans une maladie neurologique, la paraparésie spastique tropicale [3], son tropisme nerveux est très probable. Certaines des soi-disant formes sporadiques de la neurofibromatose de von Recklinghausen pourraient donc peut-être correspondre, en fait, à des infections virales par HTLV-I.

A.K.

1. Nerenberg M, Hinrichs SH, Reynolds RK Khoury G, Jay G. The tat gene of human T lymphotropic virus type I induces mesenchymal tumors in transgenic mice. Science 1987 ; 237 : 1324-9.

2. Hinrichs SH, Nerenberg M, Reynolds RK Khoury G, Jay G. Transgenic mouse mode for human neurofibromatosis. Science 1987 ; $237: 1340-3$

3. Gessain A, de Thé G. Virus HTLV-I, leucémies $\mathrm{T}$ de l'adulte et neuromyélopathies chroniques. médecine/sciences $1987 ; 3: 471-8$. 